\title{
A Space-Time Spreading Assisted Multicarrier DS-CDMA System Using OVSF Codes Employing Adaptive Mode Switching Thresholds and Adaptive Modulation
}

\author{
H. Wei, L-L. Yang and L.Hanzo ${ }^{1}$ \\ School of ECS, University of Southampton, SO17 1BJ, UK. \\ Tel: +44-23-8059-3125, Fax: +44-23-8059-4508 \\ Email:1h'1@ecs.soton.ac.uk, http://www-mobile.ecs.soton.ac.uk
}

\begin{abstract}
The Concept of Space-Time Spreading (STS) assisted, Adaptive Quadrature Amplitude Modulation (AQAM) aided MC DS-CDMA is introduced. The AQAM mode-switching thresholds are determined using an on-line real-time Bit-ErrorRatio (BER) based learning procedure. Four different schemes were studied, designed for maintaining a given target BER of $10^{-2}, 10^{-3}, 10^{-4}$ and $10^{-5}$ both with and without turbo channel coding. The effect of using different number of STS antennas ranging from 1 to 8 was also characterized in terms of the achievable effective throughput, characterising the associated system design trade-offs.
\end{abstract}

\section{INTRODUCTION}

Broadband mobile wireless communication systems aim for supporting a wide range of services and bit rates by employing techniques capable of achieving the highest possible spectral efficiency. For example, the system is expected to support services, ranging from low bit rate speech communications at $9.6 \mathrm{~kb} / \mathrm{s}$ to high bit rate video telephony at $2 \mathrm{Mb} / \mathrm{s}$. Furthermore, one of the basic requirements expected to be satisfied by broadband mobile wireless systems is the provision of high bit rate wireless Internet services and the delivery of highspeed multimedia services. However, the system capacity and hence the achievable data rate of the wireless system is limited by numerous factors, most severely by the bandwidth of the frequency-selective fading channel. In recent years, spacetime coding has received much attention and as a result it has become one of the most prevalent technique of combating the time-varying characteristics of the fading channel. In order to circumvent the effects of fading, the employment of transmit diversity techniques, such as Space-Time Block Coding (STBC) [1] and Space-Time Trellis Coding (STTC) [1,2] has been proposed. Both STBC and STTC incorporate channel coding, modulation, transmit diversity and optional receiver diversity. Another transmit diversity technique using no channel coding is known as Space-Time Spreading (STS) [3,4]. References $[4,5]$ demonstrate that a substantial diversity gain can be

The financial support of the EPSRC, UK and the EU in the framework of the NEWCOM, NEXWAY and PHOENIX projects is gratefully acknowledged. achieved by STS and this typically allows the throughout of the system to be increased. In [6] an attractive STBC based transmit diversity technique has been proposed for employment in W-CDMA systems.

In this contribution, we propose a novel STS assisted MultiCarrier Direct Sequence Code Division Multiple Access (MC DS-CDMA) system for supporting a wide range of bit rates with the aid of advantageously combining Quadrature Amplitude Modulation (QAM) [7] and Orthogonal Variable Spreading Factor (OVSF) based DS spreading codes [8]. Since OVSF codes exhibit variable Spreading Factors ( $S F s$ ), the transmitted bit rate can be adjusted with the aid of different $S F s$. More specifically, when the number of activated users in a cell is low, the interference level experienced is also low, which requires a low spreading gain, and hence the users' $S F s$ can be low. Given a fixed bandwidth and chip rate, this enables the users to support high bit rates in comparison to the scenario of having to use high $S F s$.

Finally, turbo coding techniques are invoked in our system for enhancing the achievable BER versus SNR performance. As the number of turbo channel decoding iterations increases, the achievable BER versus SNR performance can be improved at the cost of an increased complexity.

Therefore, the achievable integrity and the bit rate supported by this system is determined by the following factors:

- The specific value of the $S F s$ used, which is determined by the number of users supported within the cell considered;

- The number of transmitter antennas;

- The number of bits conveyed by the QAM scheme used on each subcarrier, which is determined by the corresponding channel transfer factor of the channel at the specific subcarrier's frequency and the corresponding transmitted power;

- The number of turbo decoding iterations, which is determined by the affordable complexity.

This contribution is organized as follows. Section 2 describes the schematic of this system. Section 3 describes two different adaptive modulation schemes, while Section 4 characterizes the performance of the STS assisted MC DS-CDMA 


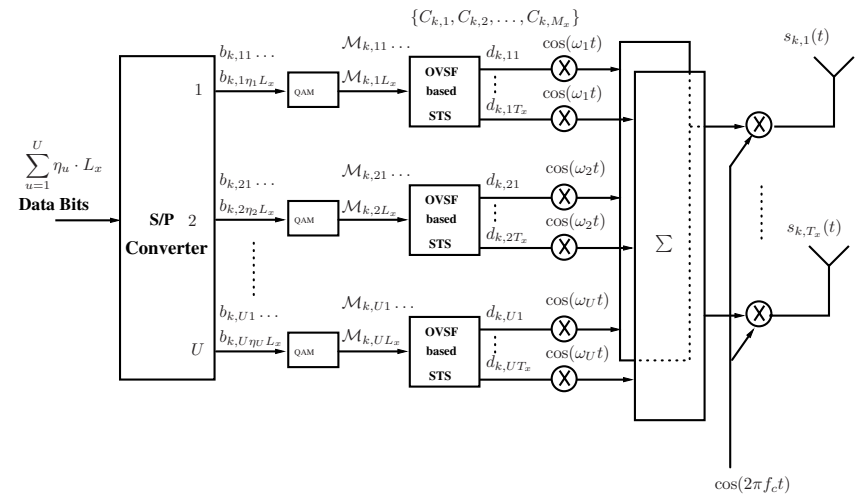

Figure 1: Schematic of the transmitter

system. Finally, Section 5 offers our conclusions and outlines our future work.

\section{SYSTEM OUTLINE}

The system considered in this contribution is a STS [4] assisted MC DS-CDMA scheme [4] using $U$ number of subcarriers, and the space-time spreading scheme has $T_{x}$ transmitter antennas, but only a single receiver antenna is invoked. Furthermore, in this contribution a synchronous MC DS-CDMA scheme designed for the downlink channel is investigated, where $K$ user signals are transmitted synchronously. The transmitter schematic of the $k$ th user is shown in Figure 1, where the transmission of complex data symbols using QAM modulation and realvalued spreading [4] were considered. As shown in Fig.1, at the transmitter a block of $\sum_{u=1}^{U} \eta_{u} \cdot L_{x}$ number of data bits having a bit duration of $T_{b}$ is S-P converted to $U$ parallel subblocks, where $\eta_{u}$ is the number of Bits Per Symbol (BPS) transmitted by the QAM scheme on the $u$ th subcarrier and $L_{x}$ is the number of QAM symbols per space-time spreading sub-block. For example, if BPSK and 64QAM are invoked on the $u$ th subcarrier, the corresponding value of $\eta_{u}$ will be 1 and 6 , respectively. Hence, each space-time spreading sub-block has $\eta_{u} L_{x}$ data bits, generating $L_{x}$ modulated symbols given by $\left\{\mathcal{M}_{k, u 1}, \ldots \mathcal{M}_{k, u L_{x}}\right\}$. These modulated symbols are space-time spread using the schemes of [3,4] with the aid of $M_{x}$ number of OVSF spreading codes, for example by $\left\{c_{k, 1}(t), \ldots, c_{k, M_{x}}(t)\right\}, k=1,2, \ldots, K$ and mapped to $T_{x}$ number of transmitter antennas. Since the bits to be transmitted are mapped to the multi-bit QAM symbols as well as to $T_{x}$ number of transmit antennas, the symbol duration of the STS signals is expanded to $U L_{x} T_{b}$, and the number of chips of the OVSF codes becomes $U L_{x} T_{b} / T_{c}=U L_{x} N$, where we have $N=T_{b} / T_{c}$ and $T_{c}$ represents the chip-duration of the orthogonal spreading codes. The orthogonal codes assume the form of $c_{k, i}(t)=\sum_{j=0}^{U L_{x} N-1} c_{k, i}[j] P_{T_{c}}\left(t-j T_{c}\right)$, where $c_{k, i}[j] \in$
$\{+1,-1\}$ and they obey the relationship of $\sum_{l=0}^{U L_{x} N} c_{i, m}[l] c_{j, n}[l]=$ 0 , whenever we have $i \neq j$ or $m \neq n$. Furthermore, $P_{T_{c}}(t)$ represents the chip waveform defined over the interval of $\left[0, T_{c}\right)$. Since the maximum available number of the OVSF codes having $U L_{x} N$ chips is $U L_{x} N$ and each user requires $M_{x}$ number of OVSF codes, the maximum number of users supported by these orthogonal codes is $\mathcal{K}_{\max }=U L_{x} N / M_{x}$. As seen in Fig.1, following STS, each STS block generates $T_{x}$ number of parallel signals to be mapped to the $T_{x}$ number of transmitter antennas. Finally, as shown in Fig.1, the Inverse Fast Fourier Transform (IFFT) is invoked for carrying out multicarrier modulation [4], and the IFFT block's output signal is transmitted using one of the transmitter antennas.

The $k$ th user's transmitted signal corresponding to the $T_{x}$ transmitter antennas can be expressed in a complex form as

$$
\mathbf{s}_{k}(t)=\sqrt{\frac{2 E_{b}}{U T_{b}} \frac{1}{M_{x} T_{x}}}\left[\mathbf{C}_{k} \mathbf{B}_{k}\right]^{T} \mathbf{w} \times \cos \left(j 2 \pi f_{c} t\right),
$$

where $E_{b} / U T_{b}$ represents the transmitted power per subcarrier expressed as $L_{x} E_{b} / U L_{x} T_{b}=E_{b} / U T_{b}$, while the factor of $M_{x} T_{x}$ represents STS using $M_{x}$ orthogonal OVSF codes and $T_{x}$ transmitter antennas. Furthermore, $\mathbf{C}_{k}$ is a $\left(U \times U M_{x}\right)$ dimensional matrix [4] constituted by the OVSF codes, while $\mathbf{B}_{k}$ is a $\left(U M_{x} \times T_{x}\right)$-dimensional matrix $[3,4]$ mapping the $U$ sub-blocks of QAM symbols to space-time spreading symbols.

Assuming that $K$ user signals expressed in the form of (1) are transmitted synchronously over single-path Rayleigh fading channels, the received complex-valued low-pass equivalent signal can be expressed as:

$R(t)=\sum_{k=1}^{K} \sum_{g=1}^{T_{x}} \sqrt{\frac{2 E_{b}}{U T_{b}} \frac{1}{M_{x} T_{x}}}\left(\left[\mathbf{C}_{k} \mathbf{B}_{k}\right]^{T}\right)_{g} \mathbf{H w}+N(t)$,

where $\mathbf{H}=\operatorname{diag}\left\{h_{1 g} \exp \left(j \psi_{1 g}\right), \ldots, h_{U g} \exp \left(j \psi_{U g}\right)\right.$ represents the channel's complex impulse response in the context of the $g$ th antenna and the coefficients $h_{i g}, i=1,2, \ldots, U ; g=$ $1,2, \ldots, T_{x}$ in $\mathbf{H}$ are independent identically distributed (i.i.d) random variables obeying the Rayleigh distribution.

After de-spreading, the output SNR of the receiver [4] becomes $\frac{E_{b}}{N_{0}} \sum_{l=1}^{T_{x}} h_{u l}^{2}$.

\section{ADAPTIVE QAM AND STS ASSISTED MC DS-CDMA}

\subsection{Mode-Switching Threshold of AQAM}

In this section, we consider two different mode-switching threshold selection methods. First, we employ a fixed threshold based adaptive QAM (AQAM) modem mode selection algorithm, which was also used in [9], adopting the AQAM techniques of [10] which were first derived for serial single-carrier AQAM modems. It was assumed that the channel quality was constant 


\begin{tabular}{|c|c|c|c|c|c|}
\hline & NoTx & BPSK & QPSK & 16QAM & 64QAM \\
\hline $10^{-2}$ & $-\infty$ & $3.31 \mathrm{~dB}$ & $6.48 \mathrm{~dB}$ & $11.61 \mathrm{~dB}$ & $17.64 \mathrm{~dB}$ \\
\hline $10^{-4}$ & $-\infty$ & $7.98 \mathrm{~dB}$ & $10.42 \mathrm{~dB}$ & $16.76 \mathrm{~dB}$ & $26.33 \mathrm{~dB}$ \\
\hline
\end{tabular}

Table 1: Optimized AQAM mode switching levels quoted from [10], which were devised for adaptive modulation, when communicating over slowly-fading narrowband Rayleigh fading channels at target BERs of $10^{-2}$ and $10^{-4}$.

for all the symbols in a transmission burst, more specifically, that the channel's fading envelope varied slowly across the transmission burst. Under these conditions, all the transmitted symbols were modulated using the same modulation mode, chosen according to the predicted SNR. Torrance et al. [10] optimized the modem mode switching thresholds for the target BERs of $10^{-2}$ and $10^{-4}$, which was deemed appropriate for a high-BER speech system and for a low-BER data system, respectively.

The resultant SNR switching thresholds optimized for activating a given modulation mode in a slowly Rayleigh fading narrowband channel are given in Table 1 for the target BERs of $10^{-2}$ and $10^{-4}$. Specifically, a given modulation mode is selected, if the instantaneous channel SNR perceived by the receiver exceeds the corresponding switching levels shown in Table 1, depending on the target BER.

Furthermore, we considered a practically-motivated AQAM mode switching approach, which is based on the philosophy of learning the switching thresholds [11] in an on-line fashion so that the system becomes capable of maximizing the achievable throughput expressed in terms of Bits Per Symbol (BPS). This learning scheme does not utilize the thresholds designed by Torrance and Hanzo [10] using Powell's optimization, which were summarized in Table 1. The learning-based scheme also refrains from using analytically optimised switching thresholds [9], or any other assumptions concerning the operating environment. The only information required by Tang's scheme [11] is the current estimated BER $p_{e}(\gamma)$ (or the Frame Error Ratio (FER)) and the target BER $P_{t h}$ of the system. The basic philosophy is that when the current $\operatorname{BER} p_{e}(\gamma)$ is lower than the target BER $P_{t h}$, this indicates that the system is capable of improving the achievable BPS throughput and hence it will reduce the switching thresholds for the sake of maximizing the throughput. By contrast, when the current BER $p_{e}(\gamma)$ is higher than the target BER $P_{t h}$, then the system is overloaded by bit errors and hence the algorithm will increase the switching thresholds for the sake of reducing the system's throughput.

In our investigations, we considered a five-mode AQAM scheme having the threshold set of $s=\left\{s_{1}, s_{2}, s_{3}, s_{4}\right\}$ designed for BPSK, QPSK, 16QAM, 64QAM, respectively. For the sake of simplicity, we assumed that the AQAM threshold difference $\left(s_{2}-s_{1}\right),\left(s_{3}-s_{1}\right),\left(s_{4}-s_{1}\right)$ between the different modulation schemes was the same as in Torrance's scheme, and we denoted them by $\triangle_{21}, \triangle_{31}, \triangle_{41}$, respectively. In other words, once we obtained the switching threshold $s_{1}$, we knew

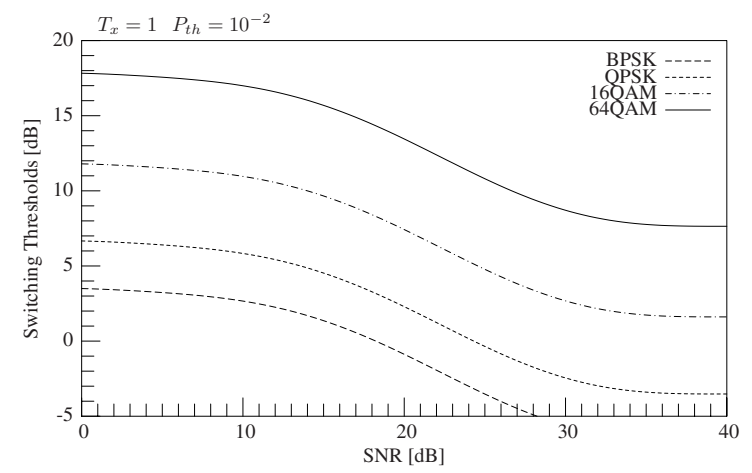

Figure 2: The learning based switching thresholds adjusted for maintaining the target BER of $P_{t h}=10^{-2}$, when using a stepsize of $\mu=0.001$.

all the switching thresholds. Similarly, once we adjusted the switching threshold $s_{1}$, all the other switching thresholds considered were adjusted. Controlling the threshold $s_{1}$ is based on the current BER performance $p_{e}(\gamma)$, where the switching thresholds can be adaptively updated as follows [11]:

$$
\begin{aligned}
& s_{1}(t)=s_{1}(t-1)+\mu \cdot \operatorname{sign}\left[p_{e}(\gamma)-P_{t h}\right] \\
& s_{2}(t)=s_{1}(t)+\triangle_{21} \\
& s_{3}(t)=s_{1}(t)+\triangle_{31} \\
& s_{4}(t)=s_{1}(t)+\triangle_{41}
\end{aligned}
$$

and where $0<\mu<1$ is the threshold control step-size. Even though the AQAM mode-switching thresholds of Table 1 were originally derived for narrow-band time-domain Rayleigh fading, they were also used for frequency-domain AQAM-aided OFDM transmissions, since the frequency domain fading is also Rayleigh distributed. Hence the algorithm is initialized using the AQAM thresholds of Table 1, and then producing the thresholds seen in Figure 2 after the completion of the initial learning phase. Perfect estimation of the current BER was assumed, although in practice the accurate estimation of the short-term 'in-burst-BER' of a transmission burst is a challenging task, as it was exemplified in [9]. This issue requires further research.

\section{PERFORMANCE OF AQAM AND STS ASSISTED MC DS-CDMA}

Figure 2 shows the evolution of the switching thresholds versus channel SNR required for maintaining the target BER of $P_{t h}=$ $10^{-2}$. We can observe that the switching thresholds are close to those proposed by Torrance and Hanzo in [10] in the SNR range of $0-20 \mathrm{~dB}$, which were summarized in Table 1 .

Figure 3 portrays the mode selection probability of the learning-based switching threshold assisted AQAM scheme. From these two figures, we can observe that as the average SNR increases, the high-order QAM modes are selected more often. 


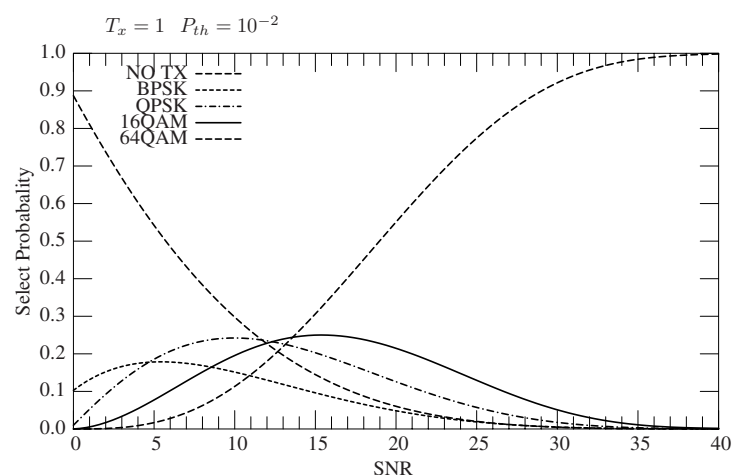

Figure 3: The mode selection probability of the learning-based switching threshold aided AQAM scheme at a target BER of $P_{t h}=10^{-2}$.

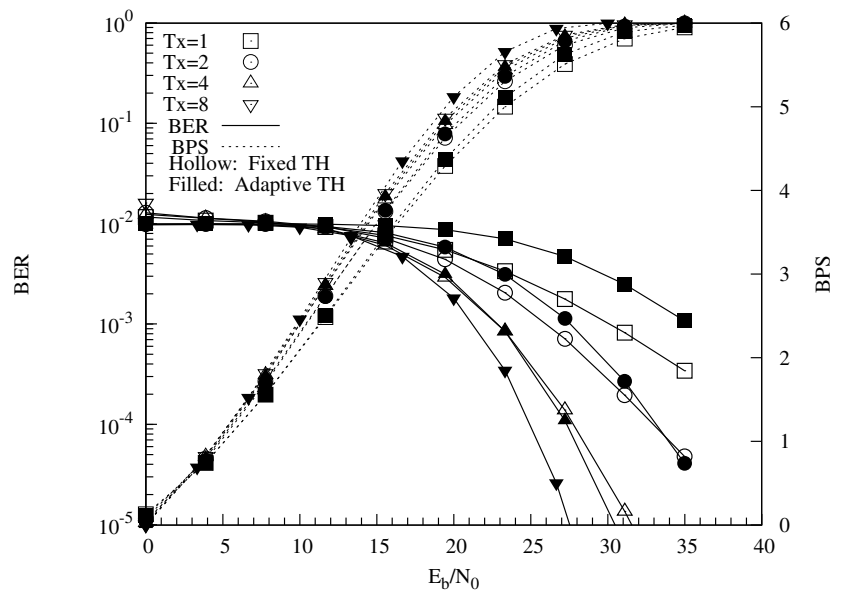

Figure 4: BER and BPS performance of AQAM aided MC DSCDMA. The fixed switching thresholds (TH) used are shown in Table 1, while the adaptive learning-based thresholds are portrayed in Figure 2, which were adjusted for maintaining the target BER of $P_{t h}=10^{-2}$. No channel coding was employed.

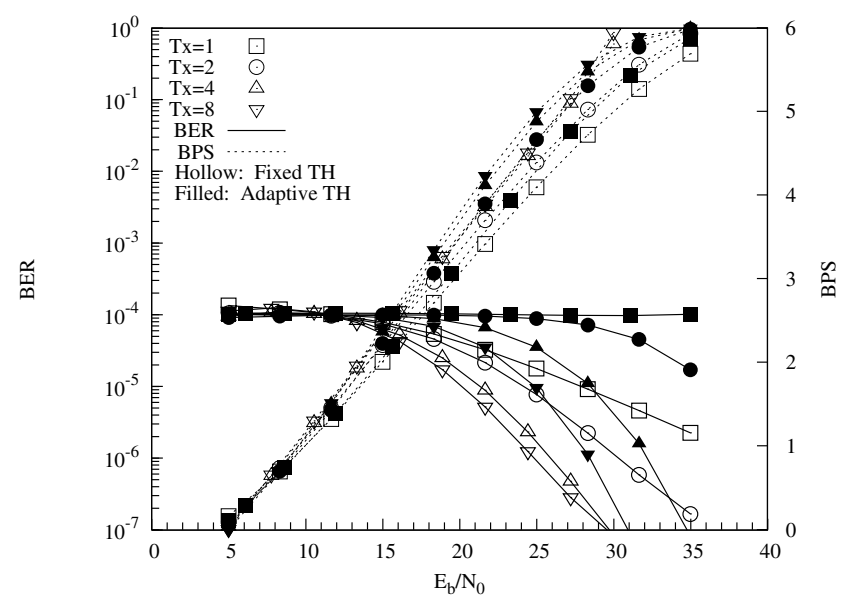

Figure 5: BER and BPS performance of AQAM aided MC DSCDMA. The fixed switching thresholds (TH) used are shown in Table 1, which were adjusted for maintaining the target BER of $P_{t h}=10^{-4}$, and no channel coding was employed.

Figure 4 shows the BER performance and BPS throughput of this system, when both the fixed switching thresholds seen in Table 1 and the adaptive thresholds of Figure 2 were employed for maintaining the target BER of $P_{t h}=10^{-2}$. By contrast, Figure 4 shows the BER performance and BPS throughput of these systems, when maintaining the target BER of $P_{t h}=10^{-4}$. From these two figures we can observe that in the high-SNR region the learning based threshold adjustment scheme exhibited a slightly better throughput than the fixed threshold based scheme of Table 1. This is because at high SNRs the switching thresholds of Figure 2 are reduced by the algorithm for the sake of increasing the achievable BPS throughput by promoting the employment of the high-throughput AQAM modes without any danger of jeopardizing the target integrity.

In Figure 6 and Figure 7 we will consider the performance of learning-based switching thresholds in conjunction with turbo coded AQAM MC DS-CDMA techniques, where the initial switching thresholds for BPSK, QPSK, 16QAM, 64QAM were $1.46 \mathrm{~dB}, 3.46 \mathrm{~dB}, 7.46 \mathrm{~dB}, 11.46 \mathrm{~dB}$, respectively. Figure 6 illustrates the achievable BER and BPS performance, when the system maintains a target BER of $10^{-3}$. By contrast, Figure 7 portrays the attainable BER and BPS performance, when maintaining a target BER of $10^{-5}$. Observe in Figure 7 that the system required an SNR of about $9 \mathrm{~dB}$ for achieving a throughput of 1 BPS, while the system of Figure 6 designed for maintaining a BER of $10^{-3}$ necessitated an SNR of only about $6 \mathrm{~dB}$ for maintaining the same throughput.

\section{CONCLUSION}

In this contribution we focussed our attention on the achievable performance of STS-assisted and AQAM-aided MC DS- 


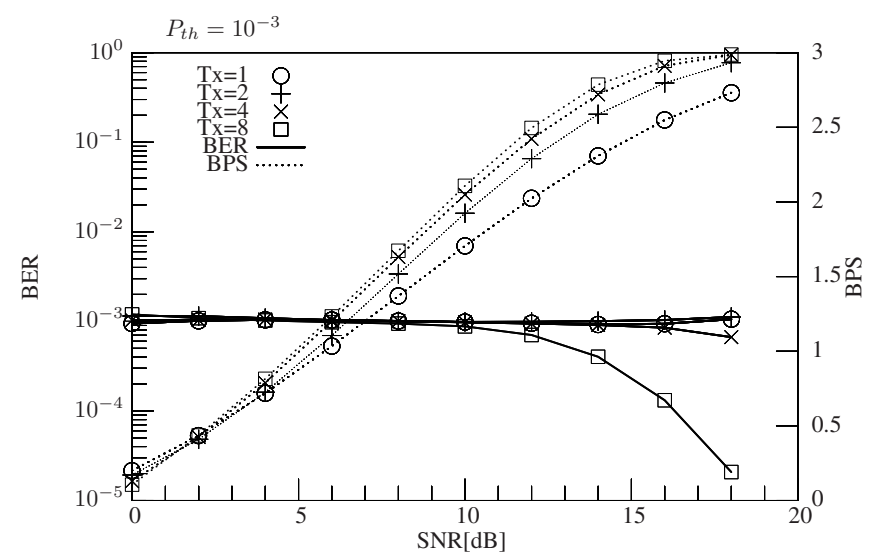

Figure 6: BER and BPS performance of AQAM aided MC DSCDMA performance in conjunction with half-rate turbo channel coding, the learning based switching thresholds, which were adjusted for maintaining the target BER of $P_{t h}=10^{-3}$,

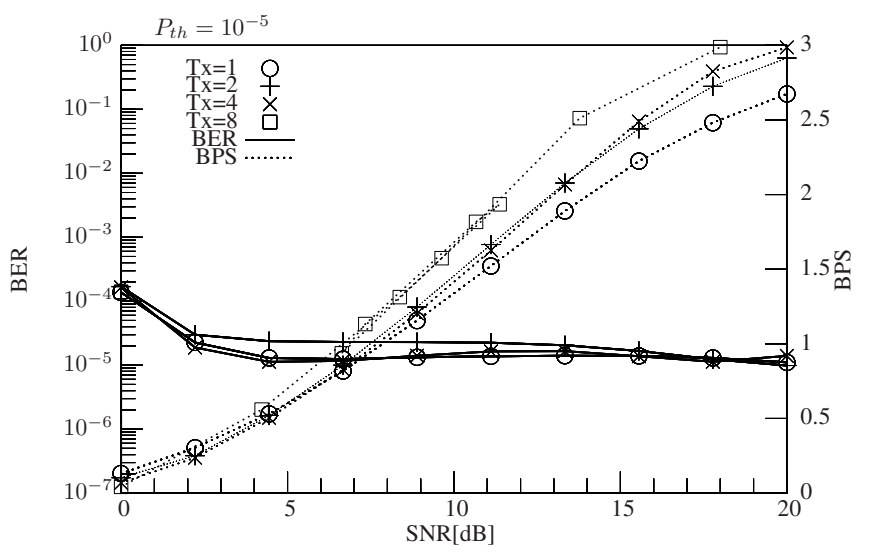

Figure 7: BER and BPS performance of AQAM aided MC DS-CDMA performance in conjunction with half-rate turbo channel coding, and the learning based switching thresholds are adjusted for maintaining the target BER of $P_{t h}=10^{-5}$.
CDMA. The system considered is capable of supporting multiple bit rates by employing OVSF spreading codes and adaptive threshold-learning assisted AQAM. It was demosntrated that as the channel quality improves, the achievable throughput is increased, while maintaining the target BER. Increasing the number of transmit antennas used by the STS scheme is beneficial in terms of reducing the channel quality fluctuations, but the associated ibncreased complexity may be more beneficially exploited by by using the extra antennas for creating additional spatially multiplexed channels, rather than for mitigating the effects of fading. Analysing these aspects in more detail constitutes the subject of our further research.

\section{REFERENCES}

[1] L. Hanzo, T. H. Liew, and B. L. Yeap, Turbo Coding, Turbo Equalisation and Space-Time Coding for Transmission over Fading Channels. John Wiley-IEEE Press, 2002.

[2] A. F. Naguib, V. Tarokh, N. Seshadri, and A. R. Calderbank, "A space-time coding modem for high-data-rate wireless communications," IEEE Journal on Selected Areas in Communications, vol. 16, pp. 1459-1478, October 1998.

[3] B. Hochwald, T. L. Marzetta, and C. B. Papadias, "A Transmitter diversity scheme for wideband CDMA systems based on Space-time spreading," IEEE Journal on Selected Areas in Communications, vol. 19, pp. 48-60, January 2001.

[4] L. Hanzo, L. L. Yang, E. L. Kuan, and K. Yen, Single- and Multi-Carrier DS-CDMA. John Wiley and IEEE Press, 2003, 1060 pages.

[5] S. M. Alamouti, "A simple transmit diversity technique for wireless communications," IEEE Journal on Selected Areas in Communications, vol. 16, pp. 1451-1458, October 1998.

[6] Proposed TDOC: 662/98 to ETSI SMG2 UMTS Standards, Space-time block coded transmit antenna diversity for WCDMA, December 1998.

[7] L. Hanzo, W. Webb, and T. Keller, Single- and Multi-carrier Quadrature Amplitude Modulation. John Wiley \& Sons, Ltd, 2000.

[8] E. H. Dinan and B. Jabbari, "Spreading Codes for Direct Sequence CDMA and Wideband CDMA Cellular Networks," IEEE in Communications Magzine, pp. 48-54, Sept 1998.

[9] L. Hanzo, C. H. Wong, and M. S. Yee, Adaptive Wireless Transceiver. John Wiley, 2001.

[10] J. Torrance and L. Hanzo, "Optimisation of switching levels for adaptive modulation in a slow Rayleigh fading channel," Electronics Letters, vol. 32, pp. 1167-1169, 20 June 1996.

[11] C. Tang, "An Intelligent Learning Scheme for Adaptive Modulation," In Proceedings of the IEEE Vehicular Technology Conference, pp. 718-719, Oct 2001. 